\title{
Intratumoral Genetic Heterogeneity
}

National Cancer Institute

\section{Source}

National Cancer Institute. Intratumoral Genetic Heterogeneity. NCI Thesaurus. Code C120464.

A heterogeneous distribution of mutations within a single neoplasm. 